\title{
Novo século, velho problema: tendência da mortalidade infantil e seus componentes no Nordeste brasileiro
}

\section{New century, old problems: infant mortality trend and its components in the northeast region of Brazil}

\author{
Carlos Dornels Freire de Souza ${ }^{1 *}\left(\mathbb{C}\right.$, Aldenyeslle Rodrigues de Albuquerque ${ }^{1}(\mathbb{C}$,

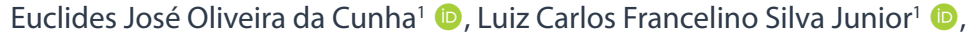 \\ José Victor de Mendonça Silva' (1D), Franklin Gerônimo Bispo Santos ${ }^{1}$ (D), \\ Maria Deysiane Porto Araújo' (D), Michael Ferreira Machado' (D), Victor Santana Santos² (D), \\ Clódis Maria Tavares 3 (D), Mônica Avelar Figueiredo Mafra Magalhães ${ }^{4}$ (D) \\ ${ }^{1}$ Departamento do curso de Medicina, Universidade Federal de Alagoas - Arapiraca (AL), Brasil. \\ ${ }^{2}$ Departamento do curso de Enfermagem, Universidade Federal de Alagoas - Arapiraca (AL), Brasil. \\ ${ }^{3}$ Escola de Enfermagem e Farmácia, Universidade Federal de Alagoas - Maceió (AL), Brasil. \\ ${ }^{4}$ Instituto de Comunicação e Informação Científica e Tecnológica em Saúde, Fundação Oswaldo Cruz - Rio de Janeiro (RJ), Brasil.
}

Como citar: Souza CDF, Albuquerque AR, Cunha EJO, Silva Junior LCF, Silva JVM, Santos FGB, Araújo MDP, Machado MF, Santos VS, Tavares CM, Magalhães MAFM. Novo século, velho problema: tendência da mortalidade infantil e seus componentes no Nordeste brasileiro. Cad Saúde Colet, 2021;29(1):133-142. https://doi.org/10.1590/1414-462X202129010340

\section{Resumo}

Introdução: A mortalidade infantil ainda representa um desafio para os países em desenvolvimento. Objetivo: Analisar a tendência da mortalidade infantil e seus componentes nos estados do Nordeste brasileiro entre 2001 e 2015. Método: Estudo ecológico envolvendo quatro indicadores de mortalidade infantil: geral, neonatal precoce, neonatal tardia e pós-neonatal. Foi aplicado o modelo de regressão por pontos de inflexão (joinpoint regression). A tendência foi classificada em crescente, decrescente ou estacionária. Calculou-se o Percentual de Variação Anual (APC, Annual Percent Change), considerando Intervalo de Confiança de 95\%. Resultados: Foi observada tendência decrescente da mortalidade infantil geral no Nordeste (-3,9\%) e em todos os estados, sendo Pernambuco com maior redução (-5,2\%). Na mortalidade neonatal precoce, somente o Maranhão apresentou tendência estacionária (-0,2\%). Na mortalidade neonatal tardia, Maranhão, Piauí, Paraíba e Sergipe apresentaram padrão estacionário. A mortalidade pós-neonatal foi a que apresentou maior redução, tendo destaque Alagoas (-8,6\%) e Pernambuco (-7,6\%). No Nordeste, esse componente apresentou variação anual de $-6,1 \%$. A partir do final da primeira década, a mortalidade pós-neonatal apresentou padrão estacionário no Nordeste, destacandose Maranhão, Ceará, Rio Grande do Norte e Sergipe. Conclusão: Embora tenha sido verificada redução da mortalidade infantil no Nordeste do Brasil, o comportamento estacionário em alguns estados configura motivo de preocupação, tendo em vista que os valores ainda são muito elevados quando comparados aos de locais desenvolvidos. Palavras-chave: mortalidade infantil; estudos de séries temporais; vigilância em saúde pública; estudos ecológicos; epidemiologia.

\footnotetext{
Abstract

Correspondência: Carlos Dornels Freire de Souza. E-mail: carlos.freire@arapiraca.ufal.br

Fonte de financiamento: nenhuma.

Conflito de interesses: nada a declarar.

Recebido em: Jul. 13, 2018. Aprovado em: Jan 20, 2020
}

Background: Infant mortality still represents a challenge for developing countries. Objective: To assess the infant mortality trend and its components in the Brazilian Northeast states between 2001 and 2015. Method: Ecological study consisting of four indicators of infant mortality were analyzed as following: general, early neonatal, late neonatal and post-neonatal mortalities. A regression analysis was applied to fit the inflection point (joinpoint regression). The trends were classified as increasing, decreasing or stationary. The Annual Percent Change (APC) was calculated considering a 95\% confidence interval.

Este é um artigo publicado em acesso aberto (Open Access) sob a licença Creative Commons Attribution, que permite uso, distribuição e reprodução em qualquer meio, sem restrições desde que o trabalho original seja corretamente citado. 
Results: Decreasing trend of overall infant mortality in the Northeast region (-3.9\%) could be measured; the state of Pernambuco showed the greatest reduction (-5.2\%). Regarding the early neonatal mortality, only the state of Maranhão showed stationary trends $(-0.2 \%)$. With respect to the late neonatal mortality, the states of Maranhão, Piauí, Paraíba and Sergipe showed stationary pattern. The post-neonatal mortality presented the greatest reduction, being highlighted the values of this significant reduction in the states of Alagoas (-8.6) and Pernambuco (-7.6). Considering the entire region, this component showed an APC of $-6.1 \%$. At the end of the first decade, post-neonatal mortality showed a stationary pattern over the Northeast region, with the highest values in the states of Maranhão, Ceará, Rio Grande do Norte and Sergipe. Conclusion: Although it has been a reduction in infant mortality in Northeastern Brazil, the stationary pattern in some states produced reasons for concern, considering that rates are still very elevated when compared to developed areas.

Keywords: infant mortality; studies of temporal statistical analysis; public health vigilance; ecological studies; epidemiology.

\section{INTRODUÇÃO}

A mortalidade infantil ainda representa um dos principais problemas de saúde pública nos países de média e baixa renda ${ }^{1,2}$, nos quais a definição de planos e políticas que visem garantir à sobrevivência das crianças tornou-se uma das mais importantes pautas no universo político e científico ${ }^{1-4}$.

Estratégias nacionais e internacionais têm gerado impactos significativos na redução da mortalidade infantil, destacando-se, no ano 2000, a definição pela Organização Mundial da Saúde (OMS) dos Objetivos de Desenvolvimento do Milênio, que apontou como meta a redução de 2/3 da mortalidade na infância entre 1990 e 2015. Vale salientar que esse objetivo leva em conta três indicadores: mortalidade em menores de cinco anos (infância), mortalidade em menores de um ano (infantil) e vacinação de crianças de até um ano de idade contra o sarampo ${ }^{5,6}$. Em 1990, a mortalidade infantil no Brasil era de 47,1/1000 nascidos vivos (NV), tendo como meta, para o ano de 2015, reduzir para 15,7/1000 NV. Quatro anos antes do prazo final, em 2011, o país apresentou mortalidade de 15,3/1000 NV, alcançando o objetivo proposto e, em 2015, a taxa de mortalidade infantil brasileira reduziu ainda mais, alcançando 13,82/1000 NV5-9.

Embora resultados favoráveis sejam evidenciados no Brasil, a taxa de mortalidade infantil não é homogênea entre as regiões. Em 2015, enquanto o Sul registrou uma taxa em torno de 10/1000 NV, no Norte o indicador alcançou 17/1000 NV. Nesse mesmo ano, o Nordeste apresentou a segunda maior taxa de mortalidade infantil, registrando 15/1000 NV. Essas assimetrias justificam a necessidade de realização de estudos sobre o tema em abordagens regionais9.

A preocupação com a taxa de mortalidade infantil justifica-se pela sua relevância, tanto pelo caráter evitáve ${ }^{8,10}$ quanto por refletirem o grau de desenvolvimento socioeconômico de determinada localidade, a infraestrutura ambiental existente e o acesso e a qualidade dos recursos disponíveis para a saúde materna e infantili, ${ }^{3,411}$.

No campo dos determinantes sociais da saúde, a mortalidade infantil é um importante indicador de monitoramento do impacto das iniquidades em saúde sobre o risco de morte das crianças. Nesse sentido, quanto maior for o indicador, maiores serão as desigualdades sociais existentes na comunidade ${ }^{3,4}$.

No campo da ação, conhecer a tendência da mortalidade infantil pode contribuir para a avaliação dos serviços de saúde, bem como subsidiar o processo de planejamento e gestão de políticas públicas e/ou planos voltados para a saúde materno-infantil ${ }^{12,13}$.

Desse modo, este trabalho objetivou analisar a tendência da mortalidade infantil e seus componentes nos estados da região Nordeste do Brasil no período de 2001 a 2015.

\section{MÉTODO}

Trata-se de um estudo ecológico de séries temporais, completando uma série história de 15 anos (2001 a 2015), realizado nos nove estados do Nordeste brasileiro: Maranhão, Piauí, Ceará, Rio Grande do Norte, Paraíba, Pernambuco, Alagoas, Sergipe e Bahia. 
Foram selecionados quatro coeficientes de mortalidade: i) mortalidade infantil geral/1000 $\mathrm{NV}$; ii) mortalidade neonatal precoce/1000 NV; iii) mortalidade neonatal tardia/1000 NV; e iv) mortalidade pós-neonatal/1000 NV. Os dados de mortalidade foram obtidos do Sistema de Informação sobre Mortalidade (SIM) e os de nascidos vivos foram obtidos do Sistema Nacional de Nascidos Vivos (Sinasc), ambos disponibilizados na plataforma do Departamento de Informática do Sistema Único de Saúde (http://datasus.saude.gov.br).

Para a análise temporal foi utilizado o modelo de regressão por pontos de inflexão (joinpoint regression model). O modelo testa se uma linha com múltiplos segmentos é estatisticamente melhor para descrever a evolução temporal dos dados do que uma linha reta ou com menos segmentos ${ }^{14}$. O joinpoint regression model permite identificar a tendência do indicador (se estacionária, crescente ou decrescente) a partir da inclinação da reta de regressão, os momentos em que ocorrem modificações no comportamento temporal (joins), a variação percentual anual (APC, Annual Percent Change) e a média de variação do período (AAPC, Average Annual Percent Change) ${ }^{14}$. Para cada tendência detectada, considerou-se o Intervalo de Confiança de 95\% (IC 95\%) e nível de significância de 5\%. Foi utilizado o software Joinpoint Regression, versão 4.5.0.1, disponibilizado pelo National Cancer Institute - USA.

Por utilizar dados secundários de domínio público e livre acesso, dispensou-se autorização do Comitê de Ética em Pesquisa.

\section{RESULTADOS}

A análise global da região Nordeste demonstrou redução significativa da mortalidade em menores de um ano (AAPC -3,9\%), decrescendo de 23,91/1000 NV em 2001 para 13,97/1000 NV em 2015. O mesmo comportamento de declínio foi verificado em cada uma das unidades federadas, sendo que o estado de Pernambuco apresentou a maior atenuação (AAPC -5,2\%), reduzindo de 26,65/1000 NV em 2001 para 13,00/1000 NV em 2015. Paralelamente, o estado de Maranhão apresentou a menor redução (AAPC -2,1\%), decrescendo de 20,15/1000 NV no início da série temporal para 15,21/1000 NV no final dela. No entanto, apesar da diminuição da mortalidade infantil observada entre 2001 e 2015, Rio Grande do Norte e Pernambuco expressaram tendências estacionárias a partir de 2009, Sergipe a partir de 2010 e Alagoas a partir de 2011 (Figura 1 e Tabela 1).

A mortalidade neonatal precoce na região Nordeste também apresentou redução significativa (AAPC -3,0\%). Apenas o estado do Maranhão apresentou tendência estacionária, apesar da leve redução da taxa de mortalidade neonatal precoce, a qual decresceu de 9,29/1000 NV em 2001 para 8,47/1000 NV em 2015 (AAPC -0,2\%), configurando-se nesse último ano como a segunda maior taxa da região Nordeste, sendo superado apenas pelo estado da Bahia (9,35/1000 NV). A maior redução foi verificada no estado de Sergipe (AAPC -5,4\%). Rio Grande do Norte e Alagoas manifestaram padrão estacionário a partir de 2010 e 2012, respectivamente (Figura 1 e Tabela 2).

Enquanto a região Nordeste apresentou declínio global da taxa de mortalidade neonatal tardia (AAPC -2,4\%), reduzindo de 2,74/1000 NV, em 2001, para 2,11/1000, em 2015, as análises segundo estado revelaram que ela se manteve estacionária no Maranhão, Piauí, Paraíba e Sergipe. Além disso, observou-se aumento da mortalidade por esse componente etário no Maranhão a partir de 2012 (APC 10,2\%) e em Sergipe a partir de 2010 (APC 5,5\%). O estado com maior redução foi o Ceará (AAPC -5,0\%) (Figura 1 e Tabela 3).

No que se refere à mortalidade pós-neonatal, houve significativa redução na taxa da região Nordeste, a qual decresceu de 9,30/1000 NV em 2001 para 3,84/1000 NV em 2015 (AAPC -6,1\%). Comportamento semelhante foi observado em todos os estados da região, destacando-se Alagoas com o maior declínio (AAPC -8,6\%), seguido do estado de Pernambuco (AAPC -7,6\%) e Paraíba (AAPC -6,3\%). No entanto, embora todos os estados tenham apresentado tendência decrescente, Maranhão, Ceará, Rio Grande do Norte e Sergipe expressaram padrão estacionário a partir do final da primeira década (Figura 1 e Tabela 4). 


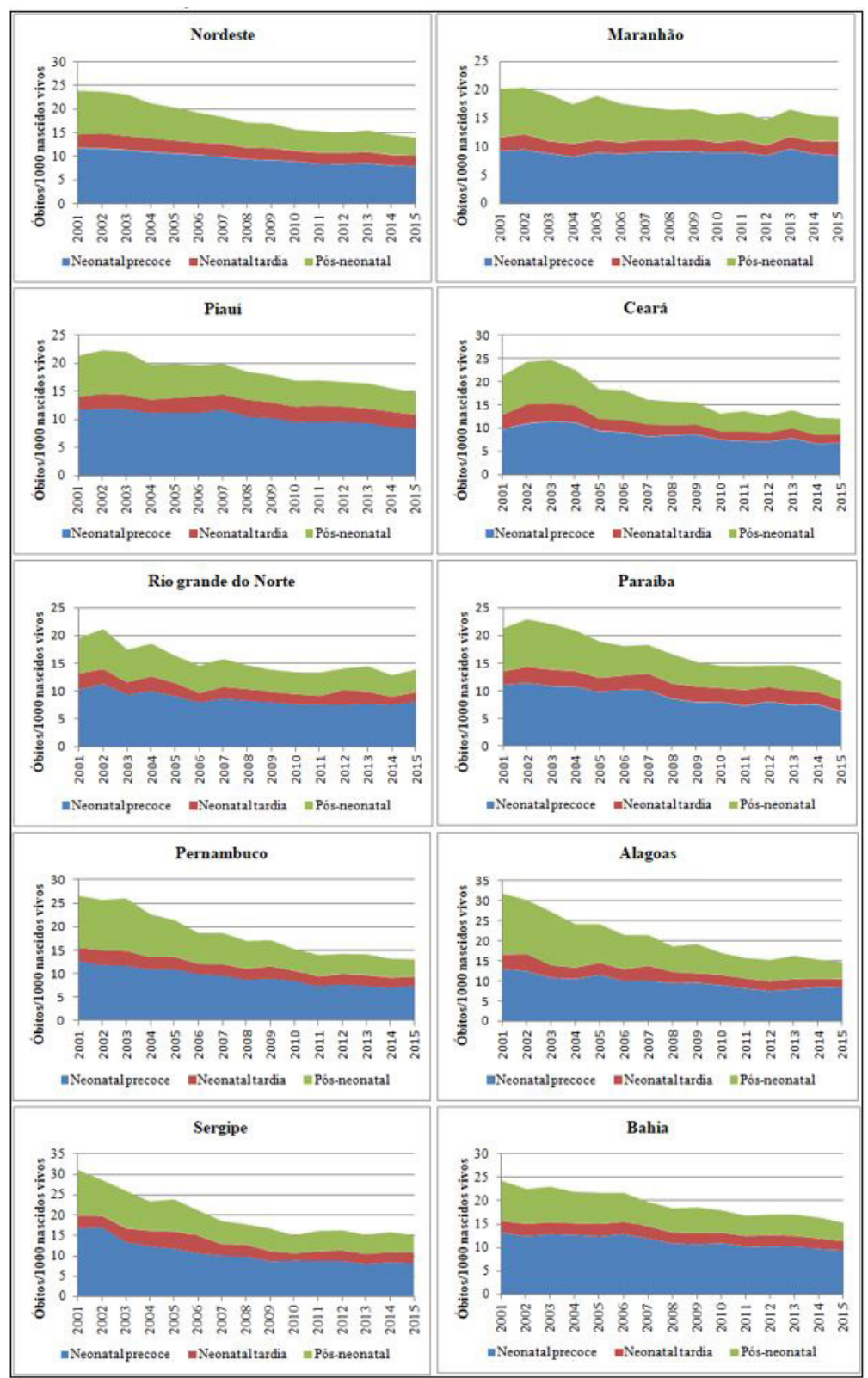

Figura 1. Evolução temporal da mortalidade infantil e seus componentes no Nordeste. Brasil, 2001-2015.

\section{DISCUSSÃO}

Assim como em outras regiões do Brasil ${ }^{1,10,15-17}$, o Nordeste vem experimentando redução substancial da mortalidade infantil e seus componentes. Tal fato pode ser explicado por importantes mudanças ocorridas no início desse novo século, sobretudo em dois eixos estruturantes. O primeiro deles refere-se às políticas de saúde, tais como o aumento da cobertura da atenção primária e do acesso ao pré-natal e aos serviços de referência; já o segundo relaciona-se com as melhorias dos determinantes sociais gerais, como educação, saneamento, moradia e renda ${ }^{7,18,19}$.

No campo das políticas de saúde, destacamos três ações importantes. A primeira diz respeito à implantação da Estratégia de Saúde da Família (ESF). Criada em 1994 [como um programa] e tornada estratégia nacional em 1998, a ESF passou a ser o mecanismo de reorganização da atenção primária à saúde no Brasil. Através de ações voltadas para a saúde materna e infantil, a ESF é apontada como um dos fatores que impactaram na redução dos 
Tabela 1. Joinpoint regression das taxas de mortalidade infantil (óbitos $<1$ ano/1000 nascidos vivos) no Nordeste. Brasil, 2001-2015.

\begin{tabular}{|c|c|c|c|c|}
\hline Região/Estado & Período & APC (IC95\%) & AAPC (IC95\%) & Classificação \\
\hline \multirow{2}{*}{ NE } & $2001-2010$ & $-4,9(-5,5 a-4,2)^{*}$ & \multirow{2}{*}{$-3,9(-4,5 \text { a }-3,2)^{*}$} & \multirow{2}{*}{ Decrescente } \\
\hline & $2010-2015$ & $-2,1(-3,8 \text { a }-0,4)^{*}$ & & \\
\hline MA & $2001-2015$ & $-2,1(-2,7 a-1,5)^{*}$ & $-2,1(-2,7 a-1,5)^{*}$ & Decrescente \\
\hline $\mathrm{PI}$ & $2001-2015$ & $-2,7(-3,1 \text { a }-2,4)^{*}$ & $-2,7(-3,1 \text { a }-2,4)^{*}$ & Decrescente \\
\hline \multirow{3}{*}{ CE } & $2001-2003$ & $7,1(-12,0$ a 30,2$)$ & \multirow{3}{*}{$-4,1(-7,4 \text { a }-0,8)^{*}$} & \multirow{3}{*}{ Decrescente } \\
\hline & $2003-2007$ & $-10,7(-19,0 a-1,5)^{*}$ & & \\
\hline & 2007-2015 & $-3,4(-5,4 a-1,3)^{*}$ & & \\
\hline \multirow[t]{2}{*}{ RN } & $2001-2009$ & $-4,8(-6,7 \text { a }-2,9)^{*}$ & \multirow{2}{*}{$-2,8(-4,3 a-1,3)^{*}$} & \multirow{2}{*}{ Decrescente } \\
\hline & 2009-2015 & $0,0(-3,1$ a 3,2$)$ & & \\
\hline PB & $2001-2015$ & $-4,3(-5,0 \text { a }-3,7)^{*}$ & $-4,3(-5,0 \text { a }-3,7)^{*}$ & Decrescente \\
\hline \multirow{2}{*}{ PE } & 2001-2009 & $-6,4(-7,2 a-5,5)^{*}$ & \multirow{2}{*}{$-5,2(-6,3 a-4,1)^{*}$} & \multirow{2}{*}{ Decrescente } \\
\hline & 2009-2015 & $-2,3(-6,0$ a 1,6$)$ & & \\
\hline \multirow{2}{*}{$\mathrm{AL}$} & $2001-2011$ & $-6,6(-7,5 a-5,7)^{*}$ & \multirow{2}{*}{$-5,1(-6,2 a-4,0)^{*}$} & \multirow{2}{*}{ Decrescente } \\
\hline & $2011-2015$ & $-1,4(-5,2$ a 2,6$)$ & & \\
\hline \multirow{2}{*}{ SE } & $2001-2010$ & $-7,4(-8,2 a-6,5)^{*}$ & \multirow{2}{*}{$-4,8(-5,6 \text { a }-3,9)^{*}$} & \multirow{2}{*}{ Decrescente } \\
\hline & $2010-2015$ & $0,0(-2,2$ a 2,3$)$ & & \\
\hline BA & 2001-2015 & $-3,2(-3,5$ a $-2,8)$ & $-3,2(-3,5 \text { a }-2,8)^{*}$ & Decrescente \\
\hline
\end{tabular}

Legenda: APC - Annual Percent Change; AAPC - Average Annual Percent Change.

*Significância estatística.

Tabela 2. Joinpoint regression das taxas de mortalidade neonatal precoce (óbitos 0 a 6 dias/1000 nascidos vivos) no Nordeste. Brasil, 2001-2015.

\begin{tabular}{|c|c|c|c|c|}
\hline Região/Estado & Período & APC (IC95\%) & AAPC (IC95\%) & Classificação \\
\hline NE & 2001-2015 & $-3,0(-3,2 \text { a }-2,7)^{*}$ & $-3,0(-3,2 \text { a }-2,7)^{*}$ & Decrescente \\
\hline MA & 2001-2015 & $-0,2(-0,7$ a 0,4$)$ & $-0,2(-0,7$ a 0,4$)$ & Estacionária \\
\hline $\mathrm{PI}$ & $2001-2015$ & $-2,4(-2,9 \text { a }-1,9)^{*}$ & $-2,4(-2,9 a-1,9)^{*}$ & Decrescente \\
\hline CE & 2001-2015 & $-3,7(-4,6 a-2,8)^{*}$ & $-3,7(-4,6 \text { a }-2,8)^{*}$ & Decrescente \\
\hline \multirow{2}{*}{$\mathrm{RN}$} & $2001-2010$ & $-3,8(-5,3 \text { a }-2,3)^{*}$ & \multirow{2}{*}{$-2,2(-3,6 \text { a }-0,8)^{*}$} & \multirow{2}{*}{ Decrescente } \\
\hline & 2010-2015 & $0,8(-2,9$ a 4,6$)$ & & \\
\hline PB & $2001-2015$ & $-3,9(-4,7 \text { a }-3,2)^{*}$ & $-3,9(-4,7 \text { a }-3,2)^{*}$ & Decrescente \\
\hline PE & 2001-2015 & $-4,3(-4,8 \text { a }-3,8)^{*}$ & $-4,3(-4,8 \text { a }-3,8)^{*}$ & Decrescente \\
\hline \multirow{2}{*}{$\mathrm{AL}$} & 2001-2012 & $-4,3(-5,3 \text { a }-3,3)^{*}$ & \multirow{2}{*}{$-2,9(-4,5 \text { a }-1,4)^{*}$} & \multirow{2}{*}{ Decrescente } \\
\hline & $2012-2015$ & $2,3(-5,2$ a 10,3$)$ & & \\
\hline \multirow{2}{*}{ SE } & 2001-2007 & $-9,2(-11,6 a-6,7)^{*}$ & \multirow{2}{*}{$-5,4(-6,6 \mathrm{a}-4,1)^{*}$} & \multirow{2}{*}{ Decrescente } \\
\hline & 2007-2015 & $-2,4(-4,1 \text { a }-0,7)^{*}$ & & \\
\hline $\mathrm{BA}$ & 2001-2015 & $-2,4(-2,9 a-2,0)^{*}$ & $-2,4(-2,9 a-2,0)^{*}$ & Decrescente \\
\hline
\end{tabular}

Legenda: APC - Annual Percent Change; AAPC - Average Annual Percent Change.

*Significância estatística. 
Tabela 3. Joinpoint regression da taxa de mortalidade neonatal tardia (óbitos 7 a 27 dias/1000 nascidos vivos) no Nordeste. Brasil, 2001-2015

\begin{tabular}{|c|c|c|c|c|}
\hline Região/Estado & Período & APC (IC95\%) & AAPC (IC95\%) & Classificação \\
\hline NE & $2001-2015$ & $-2,4(-3,0 \text { a }-1,8)^{*}$ & $-2,4(-3,0 \text { a }-1,8)^{*}$ & Decrescente \\
\hline \multirow{2}{*}{ MA } & 2001-2012 & $-2,5(-4,3 a-0,6)^{*}$ & \multirow{2}{*}{$0,1(-2,9$ a 3,2$)$} & \multirow{2}{*}{ Estacionária } \\
\hline & $2012-2015$ & $10,2(-4,5$ a 27,1$)$ & & \\
\hline \multirow{2}{*}{$\mathrm{PI}$} & $2001-2008$ & $2,7(0,4 \text { a } 5,4)^{*}$ & \multirow{2}{*}{$0,3(-1,0$ a 1,7$)$} & \multirow{2}{*}{ Estacionária } \\
\hline & $2008-2015$ & $-2,0(-4,1$ a 0,2$)$ & & \\
\hline CE & $2001-2015$ & $-5,0(-6,5 a-3,5)^{*}$ & $-5,0(-6,5 \text { a }-3,5)^{*}$ & Decrescente \\
\hline RN & $2001-2015$ & $-2,9(-5,0 \text { a }-0,8)^{*}$ & $-2,9(-5,0 \text { a }-0,8)^{*}$ & Decrescente \\
\hline \multirow{2}{*}{ PB } & 2001-2012 & $0,1(-1,4$ a 1,7$)$ & \multirow{2}{*}{$-1,9(-4,2$ a 5,0$)$} & \multirow{2}{*}{ Estacionária } \\
\hline & $2012-2015$ & $-8,8(-18,7$ a 2,2$)$ & & \\
\hline PE & $2001-2015$ & $-2,5(-3,5 a-1,5)^{*}$ & $-2,5(-3,5 a-1,5)^{*}$ & Decrescente \\
\hline \multirow[t]{2}{*}{$A L$} & $2001-2015$ & $-3,8(-5,2 \text { a }-2,4)^{*}$ & $-3,8(-5,2 \text { a }-2,4)^{*}$ & Decrescente \\
\hline & $2001-2005$ & $10,8(0,9 \text { a } 21,6)^{*}$ & \multirow{3}{*}{$-0,1(-4,1$ a 4,0$)$} & \multirow{3}{*}{ Estacionária } \\
\hline \multirow[t]{2}{*}{ SE } & $2005-2010$ & $-12,9(-20,7 \text { a }-4,4)^{*}$ & & \\
\hline & $2010-2015$ & $5,5(-1,3$ a 12,7$)$ & & \\
\hline BA & $2001-2015$ & $-1,6(-2,2 \text { a }-0,9)^{*}$ & $-1,6(-2,2 \text { a }-0,9)^{*}$ & Decrescente \\
\hline
\end{tabular}

Legenda: APC - Annual Percent Change; AAPC - Average Annual Percent Change.

*Significância estatística.

óbitos de crianças no país $5^{5,20,21}$. Um incremento de $10 \%$ da cobertura de saúde da família implicaria, por exemplo, em redução de $4,5 \%$ da mortalidade infanti ${ }^{21}$. Recentemente, estudo conduzido no semiárido brasileiro mostrou impacto positivo da ESF na redução da mortalidade de crianças, produto da ampliação do acesso a serviços de qualidade para as famílias adstritas ${ }^{22}$.

O cenário de luta pela consolidação do Sistema Único de Saúde (SUS) ganhou novo fôlego com a implementação da Política Nacional de Atenção Básica (PNAB) em 2006, e reformulada em 2011 e 2017. Alicerçada nos princípios da universalidade, integralidade e equidade, a PNAB consolidou a ESF como porta de entrada principal do SUS e instrumento de transformação da realidade sanitária, sobretudo pela aproximação que ela estabelece com as comunidades locais ${ }^{23}$.

A segunda grande iniciativa que impactou na mortalidade infantil foi a estruturação, no ano de 2011, da Rede Cegonha (RC), por meio da Portaria n 1.459 do Ministério da Saúde, com o objetivo de ampliar o acesso e melhorar a qualidade da atenção pré-natal, a assistência ao parto e ao puerpério, bem como ofertar assistência à criança com até 24 meses de vida ${ }^{24}$.

A RC está inserida no âmbito da discussão da Rede de Atenção em Saúde (RAS), cujo objetivo é promover a integração das ações e serviços de saúde para possibilitar uma atenção eficiente e de qualidade em todos os pontos de atenção, com foco na melhoria dos indicadores de morbimortalidade materno infantil. A RC é organizada a partir de quatro componentes: prénatal, parto e nascimento, puerpério e atenção integral à saúde da criança, e sistema logístico garantidor dos outros três componentes ${ }^{24}$.

Uma atenção adequada durante o pré-natal pode viabilizar tanto o diagnóstico de doenças quanto ações preventivas capazes de reduzir a ocorrência de uma série de complicações. Nesse campo da prevenção, tem destaque o desenvolvimento de práticas de cunho educativo, visando diminuir as condutas de risco e contribuindo para a redução da mortalidade tanto infantil quanto materna ${ }^{10,25}$. 
Tabela 4. Joinpoint regression da taxa de mortalidade pós-neonatal (óbitos 28 a 364 dias/1000 nascidos vivos) no Nordeste. Brasil, 2001-2015.

\begin{tabular}{|c|c|c|c|c|}
\hline Região/Estado & Período & APC (IC95\%) & AAPC (IC95\%) & Classificação \\
\hline \multirow{2}{*}{ NE } & $2001-2010$ & $-7,9(-9,1 \text { a }-6,7)^{*}$ & \multirow{2}{*}{$-6,1(-7,2 \mathrm{a}-5,0)^{*}$} & \multirow{2}{*}{ Decrescente } \\
\hline & $2010-2015$ & $-2,8(-5,7$ a 0,3$)$ & & \\
\hline \multirow{2}{*}{ MA } & 2001-2010 & $-6,5(-8,1$ a $-4,8)$ & \multirow{2}{*}{$-5,0(-6,6 a-3,4)^{*}$} & \multirow{2}{*}{ Decrescente } \\
\hline & 2010-2015 & $-2,2(-6,4$ a 2,1$)$ & & \\
\hline \multirow{2}{*}{$\mathrm{PI}$} & $2001-2008$ & $-6,4(-8,4 \text { a }-4,4)^{*}$ & \multirow{2}{*}{$-4,7(-5,9 a-3,4)^{*}$} & \multirow{2}{*}{ Decrescente } \\
\hline & $2008-2015$ & $-2,9(-4,9 \text { a }-0,8)^{*}$ & & \\
\hline \multirow{3}{*}{ CE } & $2001-2003$ & $0,5(-13,9$ a 17,3$)$ & \multirow{3}{*}{$-6,1(-8,3 \text { a }-3,9)^{*}$} & \multirow{3}{*}{ Decrescente } \\
\hline & $2003-2010$ & $-10,5(-12,8 a-8,1)^{*}$ & & \\
\hline & 2010-2015 & $-2,4(-5,7$ a 1,1$)$ & & \\
\hline \multirow{2}{*}{$\mathrm{RN}$} & 2001-2009 & $-6,4(-8,7 a-4,0)^{*}$ & \multirow{2}{*}{$-3,6(-5,5 a-1,7)^{*}$} & \multirow{2}{*}{ Decrescente } \\
\hline & 2009-2015 & $0,2(-3,7$ a 4,2$)$ & & \\
\hline PB & $2001-2015$ & $-6,3(-7,5 \text { a }-5,1)^{*}$ & $-6,3(-7,5 a-5,1)^{*}$ & Decrescente \\
\hline \multirow{3}{*}{$\mathrm{PE}$} & $2001-2003$ & $0,0(-17,4$ a 21,2$)$ & \multirow{3}{*}{$-7,6(-11,4 \text { a }-3,7)^{*}$} & \multirow{3}{*}{ Decrescente } \\
\hline & $2003-2006$ & $-15,5(-30,3$ a 2,3$)$ & & \\
\hline & 2006-2015 & $-6,5(-8,2 \mathrm{a}-4,9)^{*}$ & & \\
\hline AL & $2001-2015$ & $-8,6(-9,8 \text { a }-7,3)^{*}$ & $-8,6(-9,8 a-7,3)^{*}$ & Decrescente \\
\hline \multirow{2}{*}{ SE } & $2001-2008$ & $-9,9(-13,0 \text { a }-6,7)^{*}$ & \multirow{2}{*}{$-6,1(-8,1 \text { a }-4,0)^{*}$} & \multirow{2}{*}{ Decrescente } \\
\hline & 2008-2015 & $-2,1(-5,5$ a 1,4$)$ & & \\
\hline BA & $2001-2015$ & $-5,1(-5,9 a-4,3)^{*}$ & $-5,1(-5,9 a-4,3)^{*}$ & Decrescente \\
\hline
\end{tabular}

Legenda: APC - Annual Percent Change; AAPC - Average Annual Percent Change.

*Significância estatística.

Por meio da estruturação dessa rede de saúde, reafirmou-se o compromisso, por parte do Brasil, em garantir às crianças o direito a um nascimento seguro, bem como a um crescimento e desenvolvimento saudáveis ${ }^{24-26}$. Nesse sentido, o comportamento temporal decrescente do componente neonatal precoce nos estados do Nordeste pode ser justificado pela influência das políticas sociais e de saúde sobre os principais fatores de risco, tais como o baixo peso ao nascer, pré-natal inadequado ou não realizado e a peregrinação para o parto 4,8,27,28.

Contudo, como a competência para a execução das ações repousa sobre as unidades federativas e municípios, fragilidades têm sido apontadas, a exemplo da não efetivação, na sua totalidade, dos princípios norteadores da política, sobretudo no que diz respeito à continuidade do cuidado ${ }^{24-26}$. O aumento da mortalidade neonatal tardia em Sergipe (a partir de 2010) e no Maranhão (a partir de 2012) sugere dificuldade em manter o cuidado longitudinal para as crianças após o nascimento.

No Maranhão, por exemplo, a cobertura vacinal da população infantil, o percentual de cobertura do pré-natal e a proporção de crianças em aleitamento materno exclusivo são inferiores aos demais estados brasileiros ${ }^{29}$. Estudo conduzido nesse estado mostrou que, em 2007 , apenas $38,4 \%$ das crianças passaram por consultas de puericultura, podendo alcançar percentuais maiores nas cidades mais desenvolvidas do estado ${ }^{30}$. Esse percentual é inferior aos relatados em outras unidades federadas, como São Paulo, cuja proporção já superava $84 \%$ em $1996^{31}$. 
Investigação conduzida em municípios de pequeno e médio porte do Norte, Nordeste e Sudeste do Brasil reforça a associação da mortalidade infantil com o pré-natal inadequado e o fato de a gestante não estar vinculada à maternidade 4 . Esses achados evidenciam a necessidade de priorizar o acesso e a qualidade dos serviços assistenciais durante a gravidez, no parto e no pós-parto, o que ainda se figura como um importante desafio ${ }^{8,10}$.

A terceira iniciativa trata-se do incentivo dado ao aleitamento materno como instrumento para a redução da mortalidade infanti ${ }^{32}$. Em 1992, o Brasil aderiu à iniciativa"Hospital Amigo da Criança" das Nações Unidas para a Infância (Unicef) e da Organização Mundial da Saúde (OMS), cujo objetivo é sensibilizar e mobilizar profissionais da área hospitalar para a importância do aleitamento materno. Em 2008, foi criada a "Rede Amamenta Brasil" para fortalecer a política de incentivo ao aleitamento. O Brasil conta com uma rede envolvendo aproximadamente 326 hospitais amigos da criança, 200 bancos de leite materno e 198 postos de coleta ${ }^{33}$.

No segundo eixo estão as melhorias sociais gerais, como especial papel das políticas de transferência de renda. Nesse cenário, o Programa Bolsa Família (PBF) é apontado como um dos mais importantes determinantes da redução da mortalidade de crianças no Brasil| ${ }^{18,19,34}$, com maior impacto no componente pós-neonatal, sobretudo por evitar a ocorrência de óbitos por desnutrição e doenças diarreicas ${ }^{18}$. A análise dos primeiros cinco anos do PBF mostrou uma diminuição de $9,3 \%$ da mortalidade infantil ${ }^{18}$. Resultados semelhantes foram relatados em estudo no semiárido brasileiro 22 .

Em investigação da relação entre mortalidade infantil e condições de vida em Aracaju/ $\mathrm{SE}^{35}$, pesquisadores mostraram haver assimetrias nos padrões espaciais do evento sob a ótica das condições de vida, sendo maior nos bairros com maior carência social. Nesse cenário, as políticas de transferência de renda, a exemplo do PBF, podem reduzir essas iniquidades sociais e, desse modo, contribuir para a redução da ocorrência de óbitos infantis.

Além do desafio de reduzir a própria mortalidade infantil, é necessário destacar outro: a garantia da qualidade dos dados disponíveis nos sistemas oficiais de informações ${ }^{15}$. A qualificação das informações estatísticas através da redução das incompletudes e da correta classificação da evitabilidade possibilita a adequada compreensão do fenômeno, implicando no planejamento de ações que possam impactar diretamente nos principais determinantes da mortalidade infantil ${ }^{27}$. Um passo importante diz respeito à implantação de comitês de mortalidade infantil e fetal e sua contribuição para a qualificação das informações ${ }^{10}$.

Embora a redução observada na mortalidade infantil no Nordeste brasileiro ao longo dessas quase duas décadas do século XXI, o comportamento estacionário em alguns estados configura motivo de preocupação, tendo em vista que os valores ainda são muito elevados quando comparados aos de locais desenvolvidos. Advogamos que há um longo caminho a ser percorrido pelos estados, sobretudo no que se refere aos determinantes sociais da saúde materno-infantil. A redução das iniquidades sociais e das disparidades geográficas figura como o mecanismo mais efetivo para garantir a materialidade do direito à vida das crianças brasileiras.

\section{REFERÊNCIAS}

1. Victora CG, Aquino EM, do Carmo Leal M, Monteiro CA, Barros FC, Szwarcwald CL. Maternal and child health in Brazil: progress and challenges. Lancet. 2011;377(9780):1863-76. http://dx.doi.org/10.1016/ S0140-6736(11)60138-4. PMid:21561656.

2. Brownell M, Enns J. Reducing child mortality in high-income countries: where to from here? Lancet. 2018;391(10134):1968-9. http://dx.doi.org/10.1016/S0140-6736(18)30938-3. PMid:29731174.

3. Santos HG, Andrade SM, Silva AM, Mathias TA, Ferrari LL, Mesas AE. Avoidable causes of infant deaths due to interventions of the Brazilian Unified Health System: a comparison of two birth cohorts. Cien Saude Colet. 2014;19(3):907-16. http://dx.doi.org/10.1590/1413-81232014193.01182013. PMid:24714905.

4. Leal MC, Bittencourt SDA, Torres RMC, Niquini RP, Souza PRB Jr. Determinantes do óbito infantil no Vale do Jequitinhonha e nas regiões Norte e Nordeste do Brasil. Rev Saude Publica. 2017;51:12. http://dx.doi. org/10.1590/s1518-8787.2017051006391. PMid:28273228.

5. Instituto de Pesquisas Econômicas Aplicadas. Objetivos de desenvolvimento do milênio: Relatório Nacional de Acompanhamento [Internet]. Brasília; 2014 [citado em 2018 Fev 13]. Disponível em: http://www.ipea. gov.br/portal/index.php?option=com_content\&view=article\&id=22538 
6. World Health Organization. Levels e trends in child mortality: Report 2015 estimates developed by the UN Interagency Group for Child Mortality Estimation [Internet]. Geneva; 2015 [citado em 2018 Mar 15]. Disponível em: http://www.who.int/maternal_child_adolescent/documents/levels_trends_child_ mortality_2015/en/

7. Frias PG, Szwarcwald CL, Souza PR Jr, Almeida WS, Lira PI. Correcting vital information: estimating infant mortality, Brazil, 2000-2009. Rev Saude Publica. 2013;47(6):1048-58. http://dx.doi.org/10.1590/S003489102013000901048. PMid:24626543.

8. França EB, Lansky S, Rego MAS, Malta DC, França JS, Teixeira R, et al. Leading causes of child mortality in Brazil, in 1990 and 2015: estimates from the Global Burden of Disease study. Rev Bras Epidemiol. 2017;20(20 Suppl 1):46-60. http://dx.doi.org/10.1590/1980-5497201700050005. PMid:28658372.

9. Aquino R, de Oliveira NF, Barreto ML. Impact of the family health program on infant mortality in Brazilian municipalities. Am J Public Health. 2009;99(1):87-93. http://dx.doi.org/10.2105/AJPH.2007.127480. PMid:19008516.

10. Lisboa L, Abreu DMX, Lana AMQ, Franca EB. Mortalidade infantil: principais causas evitáveis na região Centro de Minas Gerais, 1999-2011. Epidemiol Serv Saude. 2015;24(4):711-20. http://dx.doi.org/10.5123/ S1679-49742015000400013.

11. Boing AF, Boing AC. Mortalidade infantil por causas evitáveis no Brasil: um estudo ecológico no período 2000-2002. Cad Saude Publica. 2008;24(2):447-55. http://dx.doi.org/10.1590/S0102-311X2008000200024. PMid:18278292.

12. Ramalho AA, Andrade AM, Martins FA, Koifman RJ. Tendência da mortalidade infantil no município de Rio Branco, AC, 1999 a 2015. Rev Saude Publica. 2018;52:33. http://dx.doi.org/10.11606/S15188787.2018052000280. PMid:29641657.

13. Leal MC, Swarzcwald CL, Almeida PVB, Aquino EML, Barreto ML, Barros F, Victora C. Saúde reprodutiva, materna, neonatal e infantil nos 30 anos do Sistema Único de Saúde (SUS). Ciênc. Saúde Colet. 2018;23(6):1915-1928. https://doi.org/10.1590/1413-81232018236.03942018.

14. Kim HJ, Fay MP, Feuer EJ, Midthune DN. Permutation tests for joinpoint regression with applications to cancer rates. Stat Med. 2000;19(3):335-51. http://dx.doi.org/10.1002/(SICI)1097-0258(20000215)19:3<335::AIDSIM336>3.0.CO;2-Z. PMid:10649300.

15. Ceccon RF, Bueno ALM, Hesler LZ, Kirsten KS, Portes VM, Viecili PRN. Mortalidade infantil e Saúde da Família nas unidades da Federação brasileira, 1998-2008. Cad. Saúde Colet. 2014;22(2):177-83. https:// doi.org/10.1590/1414-462X201400020011

16. Areco KC, Konstantyner T, Taddei JA. Secular trends in infant mortality by age-group and avoidable components in the State of São Paulo, 1996-2012. Rev Paul Pediatr. 2016;34(3):263-70. http://dx.doi. org/10.1016/j.rpped.2016.01.006. PMid:27105575.

17. Faria R, Santana P. Variações espaciais e desigualdades regionais no indicador de mortalidade infantil do estado de Minas Gerais, Brasil. Saude Soc. 2016;25(3):736-49. http://dx.doi.org/10.1590/s010412902016147609.

18. Shei A. Brazil's conditional cash transfer program associated with declines in infant mortality rates. Health Affairs. 2013;32(7):1274-81. http://dx.doi.org/10.1377/hlthaff.2012.0827.

19. Rasella D, Aquino R, Santos CAT, Paes-Sousa R, Barreto ML. Effect of a conditional cash transfer programme on childhood mortality: a nationwide analysis of Brazilian municipalities. Lancet. 2013;382(9886):57-64 http://dx.doi.org/10.1016/S0140-6736(13)60715-1. PMid:23683599.

20. Guanais, F. C. The combined effects of the expansion of primary health care and conditional cash transfers on infant mortality in Brazil, 1998-2010. American Am J Public Health. 2013;103(11):2000-6. http://dx.doi. org/10.2105/AJPH.2013.301452.

21. Macinko J, Guanais FC, Souza MFM. Evaluation of the impact of the family health program on infant mortality in Brazil, 1990-2002. J Epidemiol Community Health. 2006;60(1):13-9. http://dx.doi.org/10.1136/ jech.2005.038323. PMid:16361449.

22. Silva ESA, Paes NA, Silva CC. Efeitos dos programas governamentais e da fecundidade sobre a mortalidade infantil do Semiárido brasileiro. Saúde Debate. 2018;42(116):138-47. http://dx.doi.org/10.1590/01031104201811611.

23. Brasil. Ministério da Saúde. Política nacional de atenção básica [Internet]. Brasília; 2017 [citado em 2018 Jun 18]. Disponível em: http://bvsms.saude.gov.br/bvs/s audelegis/gm/2017/prt2436_22_09_2017.html.

24. Brasil. Ministério da Saúde. Portaria n 1.459/GM/MS, de 24 de junho de 2011. Institui, no âmbito do Sistema Único de Saúde (SUS), a Rede Cegonha. Diário Oficial da União [Internet]. Brasília, 24 de junho de 2011 [citado em 2018 Jun 25]. Disponível em: http://bvsms.saude.gov.br/bvs/saudelegis/gm/201 1/ prt1459_24_06_2011.html. 
25. Fernandes RZS, Vilela MFG. Estratégias de integração das práticas assistenciais de saúde e de vigilância sanitária no contexto de implementação da Rede Cegonha. Ciênc. Saúde Coletiva. 2014;19(11):4457-66. http://dx.doi.org/10.1590/1413-812320141911.21662013.

26. Pinheiro JMF, Tinoco LS, Rocha ASS, Rodrigues MP, Lyra CO, Ferreira MAF. Atenção à criança no período neonatal: avaliação do pacto de redução da mortalidade neonatal no Rio Grande do Norte, Brasil. Ciênc. Saúde Coletiva. 2016; 21(1):243-52. http://dx.doi.org/10.1590/1413-81232015211.09912014.

27. Oliveira CM, Bonfim CV, Guimarães MJB, Frias PG, Medeiros ZM. Mortalidade infantil: tendência temporal e contribuição da vigilância do óbito. Acta Paul Enferm. 2016;29(3):282-90. http://dx.doi.org/10.1590/19820194201600040.

28. Victora CG, Barros AJD, França GVA, Silva ICM, Carvajal-Velez L, Amouzou A. The contribution of poor and rural populations to national trends in reproductive, maternal, newborn, and child health coverage: analyses of cross-sectional surveys from 64 countries. Lancet. 2017;5(4):e402-e407. http://dx.doi. org/10.1016/S2214-109X(17)30077-3

29. Brasil. Ministério da Saúde. Cadernos de Informações de Saúde Informações de Saúde - Informações Gerais [Internet]. Brasília; 2011 [citado em 2018 Jun 25]. Disponível em: http://tabnet.datasus.gov.br/ tabdata/cadernos/brasil.htm

30. Cunha CLF, Silva RA, Gama MLA, Costa GRC, Costa ASV, Tonial SR. O uso de serviços de atenção primária à saúde pela população infantil em um estado do nordeste brasileiro. Cad Saude Colet. 2013;21(2):115-20. http://dx.doi.org/10.1590/S1414-462X2013000200003.

31. Monteiro RA, Schmitz BAS. Principais causas básicas da mortalidade infantil no Distrito Federal, Brasil: 1990 a 2000. Rev. Bras. Saúde Matern. Infant., 2004;4(4):413-21.

32. Jones G, Steketee RW, Black RE, Bhutta ZA, Morris SS, and the Bellagio Child Survival Study Group. How many child deaths can we prevent this year? Lancet. 2003;362(9377):65-71. http://dx.doi.org/10.1016/ S0140-6736(03)13811-1. PMid:12853204.

33. United Nations Children's Fund. The State of the World's Children 2016: A fair chance for every child [Internet]. Geneva; 2016 [citado em 2018 Jun 13]. Disponível em: https://www.unicef.org/publications/ index_91711.html

34. Lourenço EC, Guerra LM, Tuon RA, Vidal-Silva SMC, Ambrosano GMB, Corrente JE, et al. Variáveis de impacto na queda da mortalidade infantil no Estado de São Paulo, Brasil, no período de 1998 a 2008. Ciênc. Saúde Coletiva. 2014;19(7):2055-62. http://dx.doi.org/10.1590/1413-81232014197.18822013.

35. Carvalho RAS, Santos VS, Melo CM, Gurgel RQ, Oliveira CCC. Desigualdades em saúde: condições de vida e mortalidade infantil em região do nordeste do Brasil. Rev Saude Publica. 2015;49:5. http://dx.doi. org/10.1590/S0034-8910.2015049004794. PMid:25741650. 